As expected, age, sex, total cholesterol, systolic blood pressure, and current smoking were independently associated with novel plaque formation. Monocyte activity was also linked to the development of new plaques; the risk of being in a higher plaque category increased by $18 \%$ for each standard deviation $\left(0.17 \times 10^{9}\right)$ increase in monocyte count. Although IMT was also an independent predictor of novel plaque formation, no relationship was shown between monocyte count and IMT.

The authors conclude that monocyte count is independently associated with novel plaque formation in plaque-free arteries, and that this might prove to be a useful means of predicting risk in the population.

Original article Johnsen SH et al. (2005) Monocyte count is a predictor of novel plaque formation: a 7-year follow-up study of 2610 persons without carotid plaque at baseline. The Tromsø Study. Stroke 36: 715-719

\section{Restoring and maintaining sinus rhythm: SAFE-T}

The double-blind Sotalol Amiodarone Atrial Fibrillation Efficacy Trial (SAFE-T) was established to compare the efficacy of sotalol and amiodarone in restoring and maintaining sinus rhythm in patients with atrial fibrillation. Results from the study have recently been published in the New England Journal of Medicine.

Bramah Singh and colleagues screened 6,582 outpatients, of whom 665 had electrocardiographically documented persistent atrial fibrillation and were receiving anticoagulants. These patients were randomized to receive sotalol $(n=261)$, amiodarone $(n=267)$, or placebo $(n=137)$.

Sinus rhythm was restored spontaneously within 28 days of starting treatment in $27.1 \%$ of those receiving amiodarone, $24.2 \%$ of those receiving sotalol, and only $0.8 \%$ of those in the placebo group. Direct-current cardioversion was performed in the remaining cases, and this was successful in approximately $70 \%$ of patients, with no significant differences between the three treatment groups.

During the follow-up period of 1-4.5 years, the median time to a recurrence of atrial fibrillation was significantly longer in patients receiving amiodarone (487 days) than in the sotalol group (74 days) or the placebo group
(6 days). Among patients with documented ischemic heart disease-approximately $25 \%$ of the study population-the median time to recurrence of atrial fibrillation was similar in those receiving amiodarone or sotalol (569 vs 428 days, $P=0.53$ ). Importantly, those who maintained sinus rhythm had significantly greater exercise capacity and higher quality of life than those who remained in atrial fibrillation at the end of the first year of study.

In summary, amiodarone and sotalol showed similar efficacy in restoring sinus rhythm in patients with atrial fibrillation, and both drugs were superior to placebo. Amiodarone appeared to be superior in maintaining sinus rhythm, however, except in patients with ischemic heart disease.

Original article Singh BN et al. (2005) Amiodarone versus sotalol for atrial fibrillation. N Engl J Med 352: 1861-1872

\section{Dual antiplatelet therapy in carotid stenosis}

Few data are available on the efficacy of combination antiplatelet therapy for the secondary prevention of stroke, and clinical trials in this area are difficult. The Clopidogrel and Aspirin for Reduction of Emboli in Symptomatic Carotid Stenosis (CARESS) trial was set up to determine whether asymptomatic microembolic signals (MES)—detected by transcranial Doppler ultrasound (TCD) - could serve as an in vivo surrogate marker of antiplatelet efficacy.

This multicenter study included patients with recently symptomatic $\geq 50 \%$ carotid stenosis. A total of 230 subjects were screened with TCD, and MES were detected in 110 cases (47.8\%); 107 of whom were randomized to clopidogrel plus aspirin $(n=51)$ or aspirin alone $(n=56)$. The TCD recordings were then repeated on days 2 and 7.

In an intention-to-treat analysis, the proportion of patients with MES on day 7 was significantly lower in the dual-therapy group that among those receiving monotherapy (43.8\% vs $72.7 \%$ ). This corresponded to a relative risk reduction of $39.8 \%(95 \% \mathrm{Cl} 13.8-58.0$, $P=0.0046)$. In both treatment groups, MES frequency per hour was reduced by approximately $60 \%$ on day 2 , compared with baseline. Seventeen patients had recurrent ipsilateral stroke or transient ischemic attacks; MES 